\title{
The role of cavitation in submicron aerosol dispersion
}

\author{
Olga Kudryashova ${ }^{1,2^{*}}$, Evgeny Muravlev $^{1}$, Boris Vorozhtsov $^{1}$ and Igor Akhmadeev ${ }^{1}$ \\ ${ }^{1}$ Institute for Problems of Chemical and Energetic Technologies, Siberian Branch of the Russian Academy of Sciences \\ (IPCET SB RAS), 659322 Biysk, Russian Federation \\ ${ }^{2}$ Tomsk State University, 634050 Tomsk, Russian Federation
}

\begin{abstract}
Here we discuss the problem of atomizing submicron aerosols by special design atomizers enabling the cavitation regime. The formation of submicron aerosol was studied using an impulse atomizer model powered by a high-energy material and an atomizer model with a special spray nozzle generating countercurrent flows. For these atomizers, the role played by cavitation in producing submicron liquid aerosols is demonstrated herein. A mathematical model is also suggested to describe the aerosol cloud genesis. The cavitation development critical pressure, outflow velocity, and the resulting droplet sizes were evaluated. The aerosol particle size and concentration were experimentally measured by optical methods. The measured disperse parameters of aerosols during the origination and propagation of the aerosol cloud resulted from the cavitation-assisted atomization of liquids are reported: the intrinsic particle diameter of water aerosol is $10 \ldots 30 \mu \mathrm{m}$ depending on the features of the atomizer designs and their operating regimes.
\end{abstract}

\section{Introduction}

There are real-world problems in generating an aerosol cloud that require droplets of smallest size and highest specific surface. For example, pharmaceutical applications need particle size reduction down to the submicron range $(0.1 \ldots 1 \mu \mathrm{m})$ as an effective option to increase the bioavailability of active pharmaceutical ingredients $[1,2]$. Generation of fine aerosols is a stage of nanoparticles synthesis [3,4]. The aerodynamic atomization has a theoretical limit on the size of the resulting droplets as a function of the jet outflow rate [5]. However, there is a possibility to produce fine aerosol even at moderate outflow velocities and energy inputs for atomization demonstrated by the example of an impulse aerosol generator powered by a high-energy material (HEM) [6]. It was shown that droplet' size could be reduced by the cavitation phenomenon in the impulse atomization as compared to aerodynamic atomization techniques.

This paper reviews both the impulse atomizer and another atomizer design considering liquid oscillations of abnormally high amplitude originate during its operation, that employs a special arch nozzle to create countercurrent flows $[7,8]$.

This study aimed at exploring the possibility to generate fine aerosols by atomizer designs enabling cavitation and to identify optimum operating conditions from the standpoint of minimum sizes of the resulting droplets.

\section{Calculations}

\subsection{Atomizer designs}

We used two designs of an atomizer in our experiments. In a hydraulic atomizer with an arch nozzle (Figure 1a), liquid flow under pressure got into the arch of the nozzle from the two sides to generate two countercurrent flows moving at the same velocity $V_{0}$. These flows interacted to invoke oscillations and the liquid then flowed out of the rectangular orifice.

In impulse atomizer powered by HEM (Figure 1b), through a fairly wide orifice at the top, which diameter was however smaller than that of the cylindrical body, the liquid ran under the pressure of gaseous detonation products of a high-energy material.

The both atomizer designs enableed the liquid cavitation regime leading to the reduction of aerosol particle size.

\subsection{Arch nozzle}

The numerical simulation of the interaction between the coaxial countercurrent flows in the arch nozzle revealed an auto-oscillation regime with a maximum amplitude at a nozzle geometry ratio of $L / d_{0}=2$ [9]. With that, the velocity amplitude was $A=V_{x} / V_{0}=5-6$, where $V_{x}$ is the maximum transverse velocity component and $V_{0}$ is the flow velocity at nozzle inlet.

The relative particle displacement when such autooscillations occur is:

Corresponding author: olgakudr@inbox.ru 


$$
Z=\frac{V_{x}}{\omega L}
$$

where $\omega=2 \pi f$ is the auto-oscillation circular frequency.

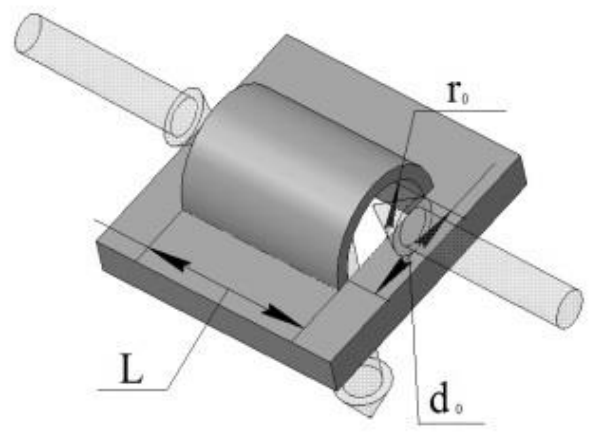

(a)

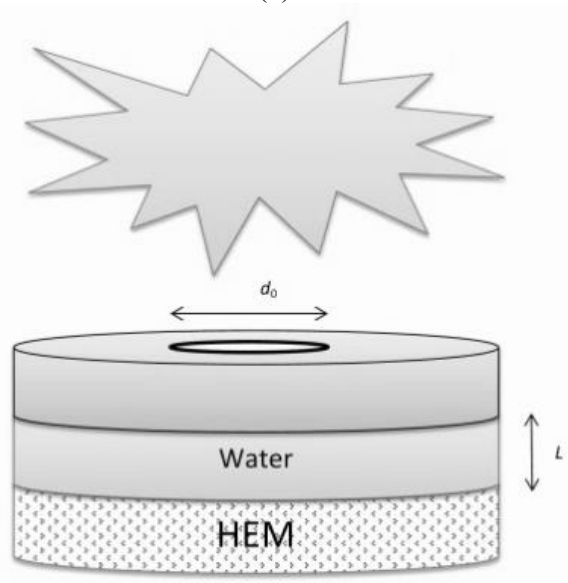

(b)

Fig. 1. (a) A schematic of the arch nozzle in the hydraulic atomizer design and (b) a schematic of the impulse atomizer wherein an explosive charge is placed at the bottom of the cylindrical body, the water bed is restrained by explosiondegradable diaphragms, and arrows show the movement of liquid flows.

The coaxial liquid flows are totally reflected from each other at the center of the arch, at the distance $L / 2$. Resonant acoustic vibrations with the wavelength $\lambda=L$ and the frequency $f=c / L$, where $c$ is the sound velocity in liquid, are determined. Expression (1) takes the form:

$$
Z=\frac{A V_{0}}{2 \pi c}
$$

Depending on hydrostatic pressure $p$ in the atomizer, the flow velocity at nozzle inlet:

$$
V_{0}=\sqrt{\frac{2\left(p-p_{0}\right)}{\rho}} .
$$

where $\rho$ is the liquid density and $p_{0}$ is the atmospheric pressure.

Under some conditions, this atomizer design provides a cavitating flow. The condition on which the cavitation regime is established is defined by the cavitation number:

$$
X=\frac{2\left(p-p_{s}\right)}{\rho V_{x}^{2}} \leq 1
$$

where $p_{s}$ is the saturated vapor pressure of the liquid.

Taking cognizance of (3), the expression for cavitation condition is written as:

$$
X=\frac{\left(p-p_{S}\right)}{A^{2}\left(p-p_{0}\right)} \leq 1
$$

otherwise, the pressure at the entrance into the system to create conditions for cavitation must be no less than the value:

$$
p \geq p_{c r}=\frac{A^{2} p_{0}-p_{s}}{A^{2}-1}
$$

\subsection{Impulse atomizer}

The aerosol generation process by using the impulse atomizer was considered earlier [6]. The triggered explosive charge produces a shock wave that translates into a series of resonant acoustic vibrations with wavelength $\lambda=L$ and frequency $f=\frac{c}{L}$. The relative amplitude of displacement in the wave is:

$$
Z=\frac{1}{2 \pi c} \sqrt{\frac{Q M_{H E M}}{M}},
$$

where $Q$ is the explosion heat of the high-energy material (HEM), $M_{H E M}$ is the HEM mass, and $M$ is the liquid mass.

The maximum pressure generated in the liquid by the exploded HEM is estimated in an approximation of the instant detonation as $p=\frac{\left(\gamma_{H E M}-1\right)}{\gamma_{H E M}} \frac{Q M_{H E M}}{V_{H E M}}$, where $V_{H E M}$ is the volume of the explosion chamber and $\gamma_{\mathrm{HEM}}$ is the adiabatic index of the detonation products.

Given contraction coefficient $\varepsilon=S_{1} / S_{2}$, where $S_{1}$ is the nozzle orifice area and $S_{2}$ is the inner cross-section area of the cylinder, the liquid outflow velocity from the atomizer is:

$$
V_{x}=\sqrt{\frac{2\left(p-p_{0}\right)}{\rho\left(1-\varepsilon^{2}\right)}} .
$$

Taking into consideration Eq. (8), expression (4) for cavitation condition takes the form:

$$
X=\frac{\left(p-p_{s}\right)\left(1-\varepsilon^{2}\right)}{\left(p-p_{0}\right)} \leq 1
$$

The condition for the critical pressure resulting from the detonated HEM to generate cavitation is: 


$$
p \geq p_{c r}=\frac{p_{0}-p_{s}\left(1-\varepsilon^{2}\right)}{\varepsilon^{2}} .
$$

\subsection{Cavitation and droplet diameter}

The both designs under question generate acoustic vibrations that create conditions for cavitation. Let us show, following the report [6], what droplet size one should expect in this case.

In the sound wave discharge phase, a breech with width $\mathrm{Z}$ emerges in the liquid, accumulating vapors. The subsequent contraction phase entails the formation of a spherical bubble with diameter $D_{c a v}$ filled with liquid vapors. The cavitation bubble size is proportional to the displacement in the wave: $D_{c a v}=k Z L$, where $k$ is the cavitation index equal to the ratio of cavitation bubbles' volume $W_{\text {allcav }}$ to liquid volume $W_{\text {liq }}: \quad k=W_{\text {allcav }} / W_{\text {liq }}$. According to data from [10], index $k$ is $0.2-0.3$ in the developed cavitation regime.

Under conditions of continuous outflow, the cavitation bubbles do not collapse but grow instead [10]. Once the cavitation bubble bearing a liquid layer gets to the atmosphere, it expands to some maximum size and then breaks down. The processes under consideration have a high speed, therefore the expansion of the cavitation bubble can be treated as adiabatic:

$$
\frac{D_{\max }}{D_{c a v}}=\left(\frac{p}{p_{\min }}\right)^{\frac{1}{3 \gamma}},
$$

where $\gamma$ is the adiabatic index of the liquid vapors, $p_{\min }$ is the minimum bubble pressure at the break-down moment, and $D_{\max }$ is the maximum bubble diameter before break-down.

The minimum bubble pressure before the bubble breaks down can be calculated from the equality relation between the internal energy of the bubble vapor and the surface energy:

$$
p_{\min } W_{\max }=\sigma S_{\max },
$$

where $W_{\max }$ is the cavitation bubble volume, $S_{\max }$ is the cavitation bubble surface area, and $\sigma$ is the surface tension, or

$$
p_{\min }=\frac{6 \sigma}{D_{\max }} .
$$

Taking account of Eqs (11) and (13), the transcendent equation is derived for estimating $D_{\max }$ :

$$
D_{\max }=D_{c a v}\left(\frac{p D_{\max }}{6 \sigma}\right)^{\frac{1}{3 \gamma}}=k Z L\left(\frac{p D_{\max }}{6 \sigma}\right)^{\frac{1}{3 \gamma}} .
$$

The bubble diameters before expansion and at the break-down moment are $D_{c a v}$ and $D_{\max }$, respectively. The outer diameter of the bubble carrying the water layer is designated as $D_{w 0}$ before expansion and as $D_{w \max }$ at the break-down moment (Figure 2).

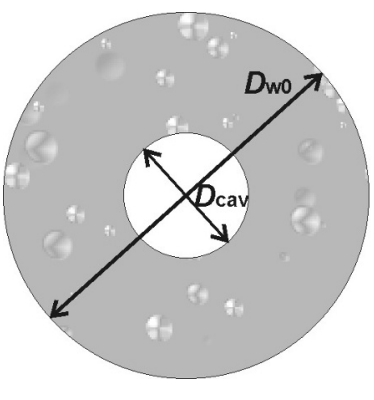

(a)

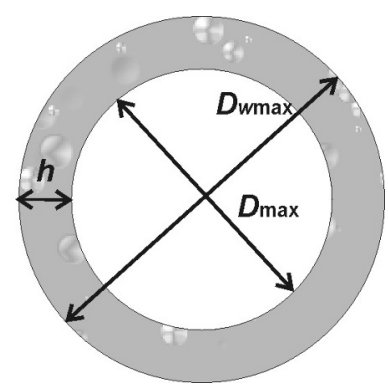

(b)
Fig. 2. The cavitation bubble carrying the water layer: (a) before expansion and (b) at the break-down moment.

The water layer thickness at the break-down moment is $h=\left(D_{w \max }-D_{\max }\right) / 2$. Taking into consideration Eq. (11) and the condition for mass equality of the liquid carried by the bubble both inside the atomizer and after flowing out of the nozzle, $D_{w 0}^{3}-D_{c a v}^{3}=D_{w \max }^{3}-D_{\max }^{3}$, the expression for $h$ is derived:

$$
h=\frac{D_{\text {cav }}}{2}\left[\left(\frac{1-k}{k}+\frac{p}{p_{\min }}\right)^{1 / 3 \gamma}-\left(\frac{p}{p_{\min }}\right)^{1 / 3 \gamma}\right] .
$$

On assumption that the minimum size of the resulting droplet is equal to the liquid layer thickness as the cavitation bubble breaks down, i.e. $D_{\text {drop }}=h$, the minimum droplet diameter is defined by Eq. (15):

$$
D_{\text {drop }}=\frac{k Z L}{2}\left[\left(\frac{1-k}{k}+\frac{p}{p_{\min }}\right)^{1 / 3 \gamma}-\left(\frac{p}{p_{\min }}\right)^{1 / 3 \gamma}\right] \text {. }
$$

To what extent the assumption of the bubble breakup into fine droplets having a diameter of about the bubble wall thickness is justified? To support the assumption, below are the pictures from the high-speed video recording of a soap bubble breakup (Figure 3). The first pictures show that the bubble bursts, for instance, into the same fragments whose diameter is comparable with the bubble wall thickness.

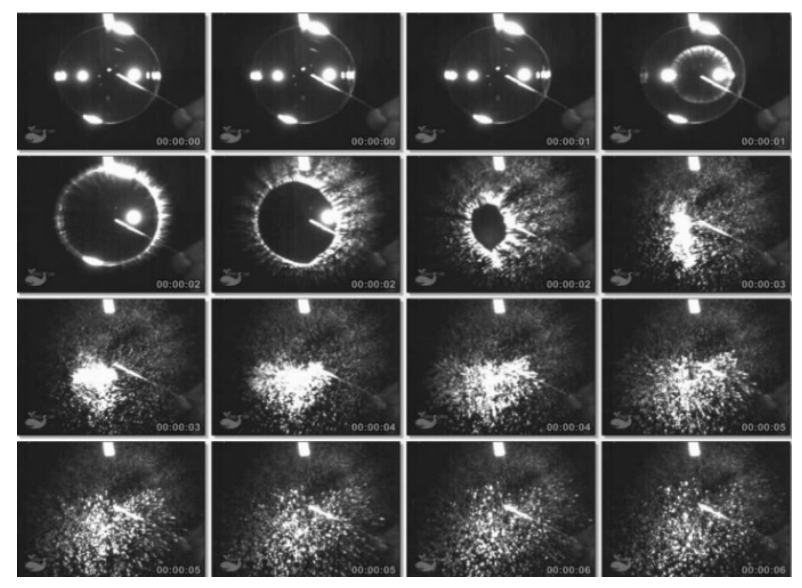

Fig. 3. (a) High-speed video recording pictures of soap bubble breakup. 
With Eqs (13) и (14) taken into account, expression (16) allows the minimum droplet diameter to be determined as a function of pressure $p$, relative displacement amplitude $Z$ in the wave, cavitated liquid layer thickness $L$, and surface tension $\sigma$. The displacement amplitude is calculated as per Eq. (2) for the arch nozzle and as per Eq. (7) for the impulse atomizer.

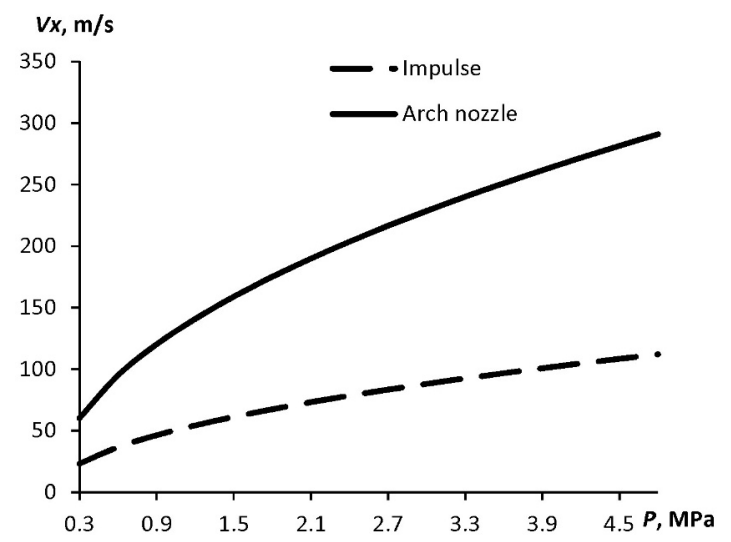

(a)

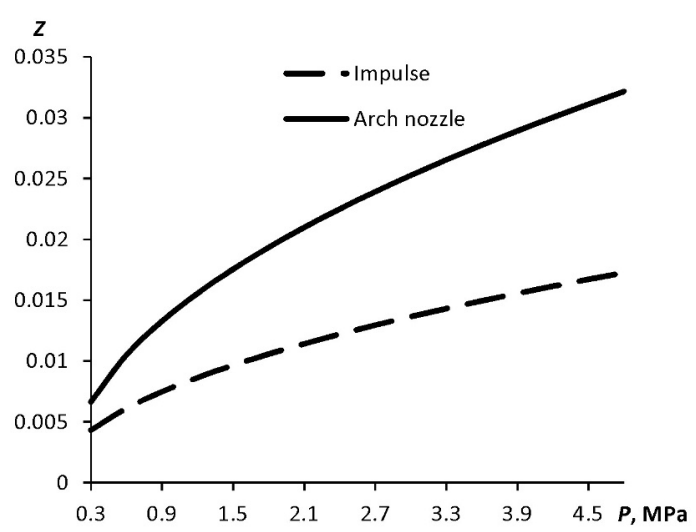

(b)

Fig. 4. (a) Outflow velocity and (b) relative displacement of liquid particles in wave plotted against pressure; velocity amplitude $A=3, L=1 \mathrm{~mm}, \varepsilon=0.5$.

Eq. (16) can estimate only the smallest possible droplet size in cavitation atomization. The atomizer pressure and cavitation index may change during the atomization (in the impulse atomizer, the pressure drops as the liquid flows out). When the bubble breaks down, the resultant droplets may happen to be a few times as large as the bubble liquid wall thickness. High-speed footage of the bubble destruction demonstrated that the resulting droplets had the order of magnitude agreeing with the bubble wall thickness, therefore the suggested equations only help estimate such magnitudes, compare atomizer designs and examine the effect of various parameters on the aerosol particle size.

The mechanism proposed herein for the formation of droplets is conceptually distinct from the well-studied mechanism consisting in the dynamic destruction of uncavitated liquid jets in nozzles and atomizers. Such a mechanism involves destruction of jets by ram air, in which case the droplet size is firstly dependent upon the jet motion velocity with respect to the air and upon liquid properties (viscosity, surface tension).

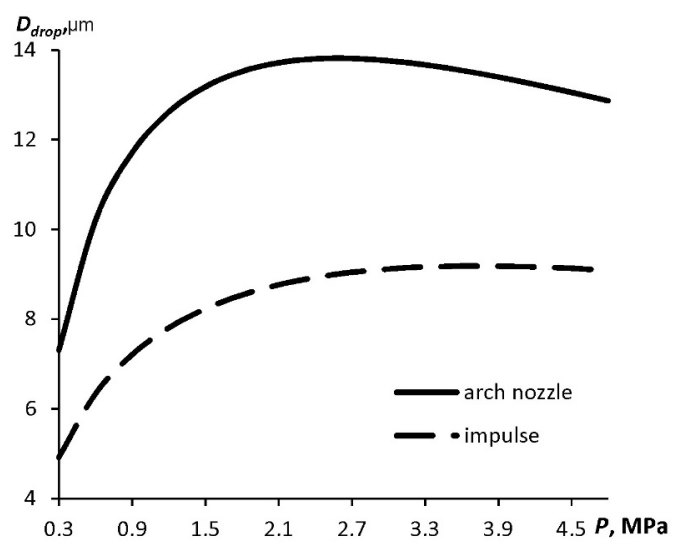

(a)

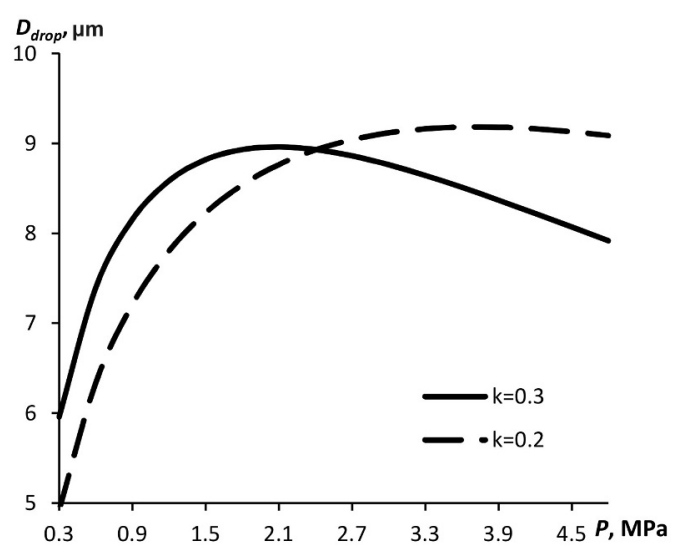

(b)

Fig. 5. (a) Minimum droplet diameter for different atomizer types and (b) different cavitation indices (impulse atomizer) plotted against pressure; velocity amplitude $A=3, L=1 \mathrm{~mm}$, $\varepsilon=0.5$.

The critical pressure depends on the saturated vapor pressure and auto-oscillation amplitude. For the arch design, there are no oscillations at $A=1$, and the cavitation does not take place. At $A \rightarrow 5$, a minimum excess of hydrostatic pressure over the atmospheric (3$5 \%$ ) is enough for successful cavitation. For the impulse atomizer, if the orifice is narrow, higher pressure levels are required for cavitation to occur because the outflow velocity is lower than that in a wider orifice.

Figure 4 illustrates the calculation result for the flow velocity and particle displacement in the wave, depending on the hydrostatic pressure. Both the flow velocity and the particle displacement in the wave increase with increasing pressure; this occurs faster in the arch nozzle (though the impulse atomizer produces higher pressure levels than the arch nozzle). As was shown above, the lower the particle displacement in the wave, the lower the droplet size, in accordance with the mathematical model suggested. Therefore the impulse atomizer should be expected to generate a finer spray than the arch nozzle system; therewith, the higher the system pressure, the greater the difference.

The estimation of the minimum droplet size plotted against pressure is displayed in Figure 5. Smaller 
droplets should be expected in the impulse atomizer under otherwise equal conditions (Figure 5a). As the relative volume of cavitation bubbles increases, the droplet size goes down (Figure 5b). The dependence of the minimum droplet diameter on pressure has a nonlinear nature, with a sharp increment at the beginning followed by a maximum and a further decrement. Thus, if the objective is to obtain the finest spray, one should strive to generate minimum pressure in the atomizer, only meeting the cavitation condition as per Eq. (6) for the arch nozzle or as per Eq. (10) for the impulse atomizer.

In non-cavitation atomizers, the pressure causes the outflow velocity to increase and the droplet size to decrease. Hence, in order to achieve a fine spray without liquid cavitation by using conventional nozzles, the pressure and the outflow velocity should be increased as maximum as possible, which entails extra power inputs. On the other hand, the typical droplet sizes resulting from atomization by conventional nozzles are dozens and hundreds of microns, even at high pressures and outflow velocities, as compared to those in the calculations presented.

\section{Experimental part}

A measuring setup has been developed whose diagram is illustrated in Figure 6. To record the aerosol particle outflow velocity, a VideoScan/C/G4 high-speed camera (VideoScan) was used with a frame rate ranging from 500 to $10000 \mathrm{fps}$ and an exposure of $50 \mu \mathrm{s}$ to $2 \mathrm{~ms}$.

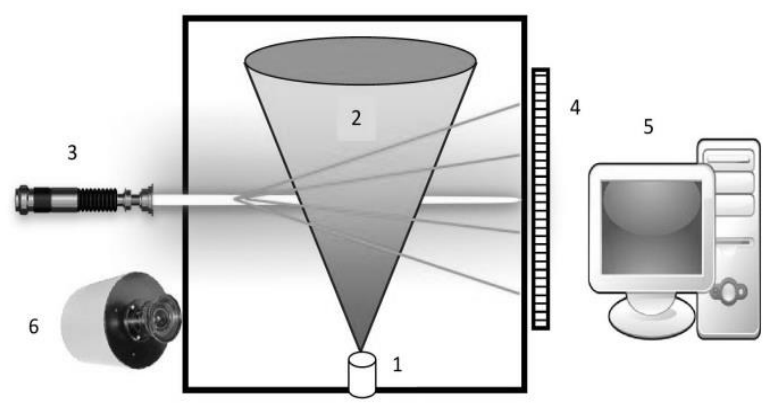

Fig. 6. A diagram of the measuring setup: 1 - atomizer, $2-$ aerosol cloud, 3 - laser, 4 - photodiode array, 5 - PC, 6 - video camera.

In the experiments, a proprietary remote method for measuring the particle concentration and the particle size distribution function was used. This method is based on the modified small-angle scattering method of probe radiation [11]. The method can measure the aerosol particle concentration and size distribution with a high time resolution. The emitter was an LG-78 He-Ne laser with a wavelength of $0.63 \mu \mathrm{m}$, whose radiation when passing through a diaphragm was modulated with $40 \mathrm{~Hz}$ to exclude the background radiation. To record the scattering radiation at the angle range $\theta=(0-15)^{\circ}$, FD$24 \mathrm{~K}$ photodiodes were employed from which a photodiode array had been made. The photodiode array used here consists of seven radiation sensors. The signals from the photodiodes were recorded on a PC fitted with an L-783 A/D converter (L-Card, Moscow) with a sampling frequency of $285 \mathrm{kHz}$ for each channel. The experiments were done with water aerosol.

\section{Results and discussions}

A chart graph of the water droplet diameter distribution obtained by using the arch nozzle under 6 atm and by using the impulse atomizer $(p=11 \mathrm{MPa})$ is displayed in Figure 7a. These experiments showed the smallest droplet size. The impulse atomizer gave a significantly higher proportion of particles having a diameter below 5 $\mu \mathrm{m}$, yet the statistical mean distributions for the both designs were similar. Figure $7 \mathrm{~b}$ shows a bar chart of the water droplet diameter distribution when atomized by the arch nozzle at different water pressures (0.6 and 1.8 $\mathrm{MPa})$. The considerable decrease in the fineness of spray with increasing pressure is on a par with the theoretical data (Figure 7a).

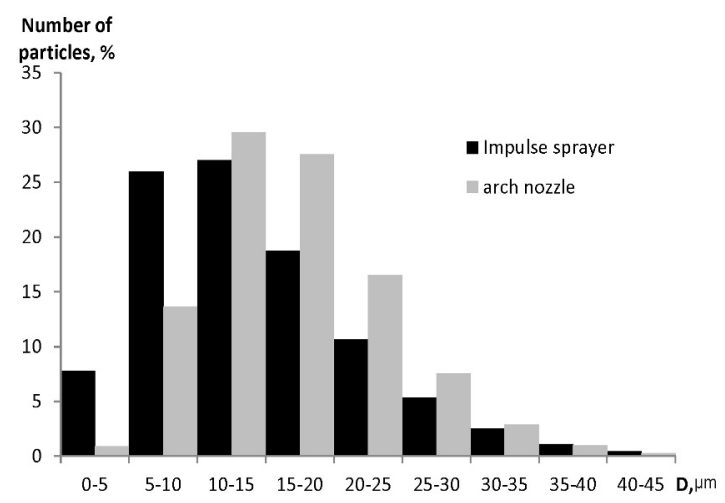

(a)

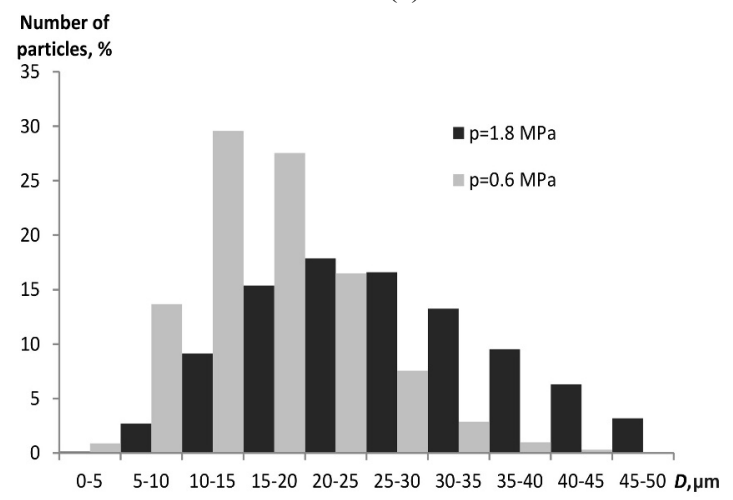

(b)

Fig. 7. Bar graphs of the water droplet diameter distribution with two atomizer types: (a) arch nozzle (grey) and impulse atomizer (black); (b) at different pressures for arch nozzle: 0.6 $\mathrm{MPa}$ (grey) and $1.8 \mathrm{MPa}$ (black).

The arithmetic mean particle diameter, $D_{10}$, as measured in the spray of the arch nozzle at different water pressures (from 0.6 to $1.8 \mathrm{MPa}$ ) and in the spray of the impulse atomizer at the two pressures of 5 and 11 $\mathrm{MPa}$, as well as calculated minimum droplet diameters as per the aforesaid mathematical model, is depicted in Figure 8.

Because the mathematical model gives a lowerbound estimate of droplet sizes, the calculated and 
experimental results can be considered satisfactory. The fineness of spray can be explained by the cavitation mechanism of droplet formation. The known mechanism for dynamic destruction of jets in the air [5] estimates droplet sizes at dozens and hundreds of microns; besides, the lower the pressure in the system, the lower the jet velocity and the larger the droplets - which is what we did not observe in the experiment.

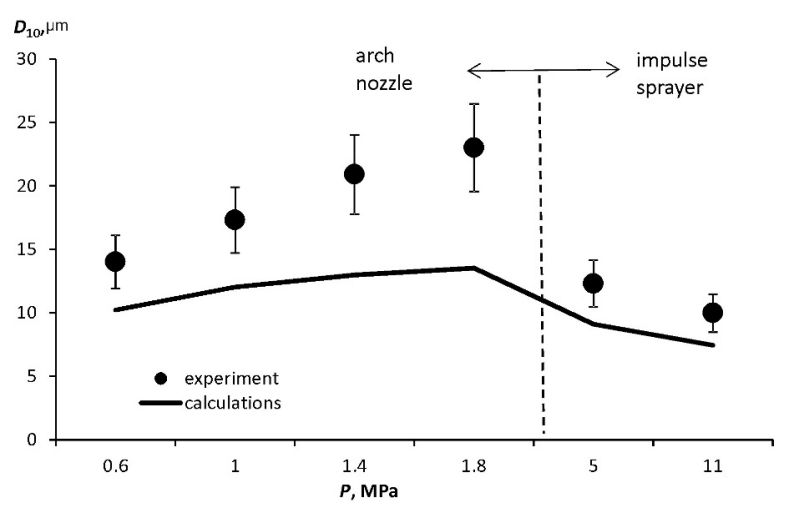

Fig. 8. Arithmetic mean diameter of droplets when atomized by two types of cavitation atomizers at different pressures: experimental (dots) and calculated minimum droplet diameters (curve).

Photographs of the spray taken by the video camera are given in Figure 9.

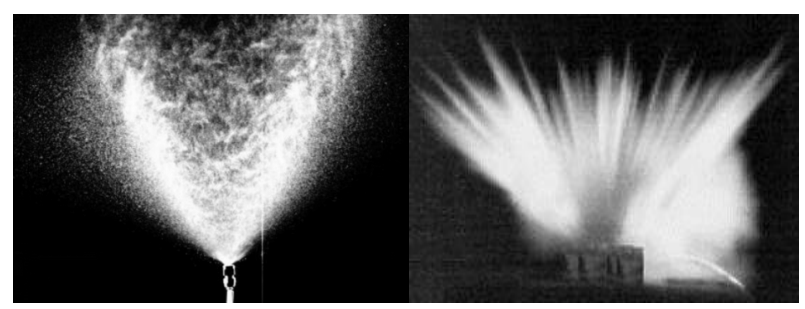

(a)

(b)

Fig. 9. (a) arch nozzle performance (0.6 MPa) and (b) impulse atomizer performance (11 MPa).

Both the experiment and the calculation allow for the conclusion that the arch nozzle provides a less dispersed spray than the impulse atomizer, with the pressure growth leading to the increased droplet size, as is seen in Figure 3 showing the $D_{d r o p}(p)$ curve with the maximum. Hydraulic spray nozzles operate at lower pressures than the impulse atomizer. Hence, for the arch spray nozzle, it can be advised to use lower pressures of a fluid when atomized, if the purpose is to reduce the droplet size. In contrast, pressures generated in the impulse atomizer powered by a high-energy material are within the downward part of the $D_{d r o p}(p)$ curve, yet the droplet size reduction within that part of the curve is not so significant. Overall, the impulse atomizer provides a finer spray than the arch nozzle device.

\section{Conclusion}

It has theoretically been shown that acoustic vibrations in the atomized medium promote the cavitation development. The cavitated liquid while flowing out into the air breaks into smaller droplets as opposed to the case of continuous fluid. The high specific surface of droplets is achieved at lower power inputs. In addition, a dimensionless parameter was derived that characterizes the cavitation process efficiency when the liquid is atomized. The experimental results from investigating the particle size of aerosols generated by the two atomizer designs demonstrate the intrinsic particle diameter of water aerosol is about $10-30 \mu \mathrm{m}$, depending on the features of the atomizer designs and their operating regimes.

This work was done using instruments of the SB RAS Biysk Regional Center for Shared Use of Scientific Equipment (IPCET SB RAS, Biysk).

\section{References}

1. R. Gorny, G. Schaldach, P. Walzel, M. Thommes, In Ilass Europe. 28th european conference on Liquid Atomization and Spray Systems, 162 (Editorial Universitat Politècnica de València, 2017)

2. R. Strob, A. Dobrowolski, G. Schaldach, P. Walzel, M. Thommes, Int. J. Pharm. 548 (1), 423 (2018)

3. K. Okuyama, I. Wuled Lenggoro, Chem. Eng. Sci., 58.3-6, 537 (2003)

4. M. Mezhericher, J. K. Nunes, J. J. Guzowski, H.A. Stone, Chem. Eng. J. 346, 606 (2018)

5. D.G. Pazhi, V.S. Galustov Fundamental Techniques of Liquid Atomization (Khimiya, Moscow, 1984)

6. O.B. Kudryashova, B.I. Vorozhtsov, E.V. Muravlev, I.R. Akhmadeev, A.A. Pavlenko, S.S. Titov, Propell. Explos. Pyrot. 36, 524 (2011)

7. V.P. Karlikov, S.L. Tolokonnikov, O.V. Trushina, Fluid Dynamics 46, 418 (2011)

8. K.A. Kuftyrev, A.N. Kolosnitsyn, Fundamental Research 4, 93 (2015)

9. D.M. Denisikhina Numerical Simulation of Autooscillatory Ventilating Flows, Monograph (SaintPetersburg, 2005)

10. V.K. Kedrinskiy Gas-dynamics of Explosion: Experiment and Models (SB RAS Press, Novosibirsk, 2000)

11. O. Kudryashova, A. Pavlenko, B. Vorozhtsov, S. Titov, V. Arkhipov, E. Maksimenko, I. Akhmadeev, E. Muravlev Remote Optical Diagnostics of Nonstationary Aerosol Media in a Wide Range of Particle Sizes, in Book: Photodetectors,( InTech, Rijeka, 2012) 